\title{
EXTENSION OF A THEOREM OF BOCHNER ON EXPRESS- ING FUNCTIONALS AS RIEMANN INTEGRALS
}

\author{
BROCKWAY MCMILLAN AND PACO LAGERSTROM
}

Introduction. S. Bochner ${ }^{1}$ has shown that an additive homogeneous functional defined over a sufficiently large class $C$ of functions can be realized as a Riemann integral with respect to a finitely additive measure $V$ in the space $X$ over which the functions are defined. His proof makes use of the fact that the constant function belongs to $C$, as a result, $V(X)$ is finite. It is the purpose of this note to show that a similar theorem holds even when $V(X)$ turns out to be infinite. A modification of Bochner's proof would suffice for this stronger theorem. We have chosen rather to treat it as a problem of extending the domain of definition of the given functional.

Throughout we have used the symbol $\rightarrow$ to be read as "implies." The equality $\equiv$ is used to denote an equality which holds by definition.

Notations. We consider a space $X$ of points $x$, and real-valued point functions $f, g, \cdots$ over $X$. Given $f, g$, and real numbers $a, b$, we shall write

$$
|f|, a f+b g, f g, f \wedge g, f \vee g, f^{+}, f^{-},
$$

respectively, for those functions whose values for each $x$ are given by

$$
\begin{aligned}
& |f(x)|, \quad a f(x)+b g(x), \quad f(x) g(x), \quad \inf [f(x), g(x)], \\
& \quad \sup [f(x), g(x)], \quad \sup [f(x), 0], \quad \sup [-f(x), 0] .
\end{aligned}
$$

We shall write $a$ for the constant function $f(x)=a$, and write $f \geqq g$ if for each $x, f(x) \geqq g(x)$. The function which coincides with $f$ on a set $A$ and is equal to 0 in $X-A$ will be denoted by $f_{A}$. In particular we write $1_{A}$ for the characteristic function of the set $A$. The symbol $\varnothing$ will denote the empty set.

It is clear that $f=f^{+}-f^{-}$, and that

$$
\left(f_{A}\right)^{+}=\left(f^{+}\right)_{A}, \quad\left(f_{A}\right)^{-}=\left(f^{-}\right)_{A} .
$$

1. $R$-measure.

1.1. By an $R$-measure in $X$ we shall mean a set function $V(E)$ defined for sets $E$ of a family $A$ with the following properties:

Received by the editors January 10, 1943, and, in revised form, September 5, 1944.

${ }^{1} \mathrm{~S}$. Bochner, Additive set functions on groups, Ann. of Math. vol. 40 (1939) pp. 769-799. The theorem in question occurs in paragraph 4. 
If $E, E_{1}, E_{2} \in \mathrm{A}$, then

(1) $E_{1} \cup E_{2} \in \mathbf{A}$,

(2) $X-E \in A$,

(3) $0 \leqq V(E) \leqq \infty$,

(4) $V(E)=0$, and $B \subset E \rightarrow B \in \mathbf{A}$,

(5) $E_{1} \cap E_{2}=\varnothing \rightarrow V\left(E_{1} \cup E_{2}\right)=V\left(E_{1}\right)+V\left(E_{2}\right)$.

Also

(6) there exists an $E \in \mathrm{A}$ with $0<V(E)<\infty$.

1.2. Remark. (1), (2) imply $E_{1} \cap E_{2} \in \mathbf{A}, E_{1}-E_{2} \in \mathbf{A}, \varnothing \in \mathbf{A}, X \in \mathbf{A}$.

2. The Riemann integral. Let $\Delta$ be the class of all partitions $\delta$ of $X$ into finitely many pairwise disjoint sets of A. Given any $f \geqq 0$, bounded on $E \in \mathbf{A}$ with $V(E)<\infty$, we define

2.1

$$
S_{u}(f, E, \delta) \equiv \sum_{D \in \delta} V(D \cap E)\left(\sup \left\{f_{E}(x) \mid x \in D\right\}\right),
$$

$$
\begin{aligned}
S_{l}(f, E, \delta) & \equiv \sum_{D \in \delta} V(D \cap E)\left(\inf \left\{f_{E}(x) \mid x \in D\right\}\right), \\
S_{u}(f, E) & \equiv \inf \left\{S_{u}(f, E, \delta) \mid \delta \in \Delta\right\},
\end{aligned}
$$$$
S_{l}(f, E) \equiv \sup \left\{S_{l}(f, E, \delta) \mid \delta \in \Delta\right\} \text {, }
$$

2.3

$$
\begin{aligned}
S_{u}(f) & \equiv \sup \left\{S_{u}(f, E) \mid E \in \mathbf{A}, V(E)<\infty\right\}, \\
S_{l}(f) & \equiv \sup \left\{S_{l}(f, E) \mid E \in \mathbf{A}, V(E)<\infty\right\} .
\end{aligned}
$$

We define the function classes

$$
\begin{aligned}
& R_{E} \equiv\left\{f \mid S_{u}\left(f^{+}, E\right)=S_{l}\left(f^{+}, E\right)<\infty,\right. \\
& \left.\qquad S_{u}\left(f^{-}, E\right)=S_{l}\left(f^{-}, E\right)<\infty\right\}, \\
& R \equiv\left\{f \mid S_{u}\left(f^{+}\right), S_{u}\left(f^{-}\right)<\infty \quad \text { and } \quad\left(V(E)<\infty \rightarrow f \in R_{E}\right)\right\} .
\end{aligned}
$$

Finally,

$$
f \in R \rightarrow \int f \equiv S_{u}\left(f^{+}\right)-S_{u}\left(f^{-}\right)
$$

It is easily shown that the supremum and infimum in 2.2 are in fact monotone limits over the directed set of partitions $\delta \in \Delta, \Delta$ being ordered by refinement. From this fact and from the definition it then follows that (when $E, E_{1}, E_{2} \in \mathbf{A}$ and $V(E), V\left(E_{i}\right)<\infty$ )

$2.6 f \geqq 0$ and $E_{1} \subset E_{2} \rightarrow 0 \leqq S_{u}\left(f, E_{1}\right) \leqq S_{u}\left(f, E_{2}\right)$,

$2.7 f \geqq 0 \rightarrow S_{u}(f, E)=S_{u}\left(f_{E}\right), \quad S_{l}(f, E)=S_{l}\left(f_{E}\right)$,

$2.8 f \in R_{E} \rightarrow\left(f_{E} \in R\right.$ and $\left.\int f_{E}=S_{l}\left(f^{+}, E\right)-S_{l}\left(f^{-}, E\right)\right)$, 
2.9 $f \in R$ and $f \geqq 0 \rightarrow \int f=\sup \left\{\int f_{E} \mid E \in A, V(E)<\infty\right\}$,

$2.91 f \in R \rightleftarrows f^{+}, f^{-} \in R$,

$2.92 \int a f+b g=a \int f+b \int g$.

\section{Modules.}

3.1. A class $C$ of real-valued functions over $X$, together with a realvalued linear functional $L$ defined over $C$, is called a module if it satisfies conditions 3.1 (1)-(11) below. $(f, g$ denote elements of $C$; $a$, a real number.)

(1) Each $f$ in $C$ is bounded.

(2) $f+g \in C$.

(3) $a f \in C$.

(4) $f \wedge 0 \in C$.

(5) $f \wedge 1 \in C$.

(6) $|L(f)|<\infty$.

(7) $L(f+g)=L(f)+L(g)$.

(8) $L(a f)=a L(f)$.

(9) $f \geqq 0 \rightarrow L(f) \geqq 0$.

(10) There exists an $f \in C$ with $L(f)>0$.

(11) $\operatorname{Inf}_{a>0} L(f \wedge a) \leqq 0$.

The main theorem of this paper is:

3.2. If $C$ is a module, there exists an $R$-measure $V(E)$ in $X$ such that (1) $C \subset R$, (2) $f \in C \rightarrow L(f)=\int f$, (3) given $e>0$ and $g \in R$, with $g \geqq 0$, there exists an $f \in C$ such that $0 \leqq f \leqq g$ and $L(f) \leqq \int g<L(f)+e$.

Before constructing the $R$-measure we prove some elementary properties of a module $C$.

3.3 $f, g \in C \rightarrow f \vee g, f \wedge g \in C$. For example,

$$
f \vee g=g-(g-f) \wedge 0 \text {. }
$$

$3.4 f \in C, a>0 \rightarrow f \wedge a \in C$, for $f \wedge a=a \cdot(1 / a) f \wedge 1$.

$3.5 f, g \in C, f \geqq g \rightarrow L(f) \geqq L(g)$, for $L(f)-L(g)=L(f-g) \geqq 0$.

$3.6 f, 1_{A} \in C \rightarrow f_{A}=f \cdot 1_{A} \in C$, for $0 \leqq f(x) \leqq b \rightarrow f_{A}=f \wedge b 1_{A}$.

4. Completion of a module. In 4.1-4.5 below, $f, h$ denote elements of a module $C$, while $g$ may be any function.

4.1. $L_{u}(g) \equiv \inf \{L(h) \mid h \geqq g\}$ (if there exists an $h$, such that $h \geqq g$ ).

4.2. $L_{l}(g) \equiv \sup \{L(f) \mid f \leqq g\}$ (if there exists an $f$ such that $f \leqq g$ ). 
4.3. $C^{*} \equiv\left\{g \mid L_{u}(g)=L_{l}(g)\right\}$.

4.4. $L^{*}(g) \equiv L_{u}(g)=L_{l}(g)$ (for $\left.g \in C^{*}\right)$.

4.5. $C \subset C^{*}$ and $L^{*}(f)=L(f)$.

4.6. $C^{*}$ is a module. We show (except for some obvious cases) that $C^{*}$ has properties (1)-(11) of 3.1 .

(3) and (8): Suppose $g \in C^{*}$ and, say, $a<0$. Then

$$
\{f \mid f \leqq a g\}=\{a h \mid h \geqq g\} \text {. }
$$

Hence

$$
L_{l}(a g)=\sup \{L(a h) \mid h \geqq g\}=a \inf \{L(h) \mid h \geqq g\}=a L^{*}(g) .
$$

Similarly

$$
L_{u}(a g)=a L^{*}(g) .
$$

(2) and (7): Suppose $g_{1}, g_{2} \in C^{*}$. Then

$$
\left\{f_{1}+f_{2} \mid f_{i} \leqq g_{i}\right\} \subset\left\{f \mid f \leqq g_{1}+g_{2}\right\} .
$$

Hence $L_{l}\left(g_{1}\right)+L_{l}\left(g_{2}\right) \leqq L_{l}\left(g_{1}+g_{2}\right)$ and, dually, $L_{u}\left(g_{1}+g_{2}\right) \leqq L_{u}\left(g_{1}\right)$ $+L_{u}\left(g_{2}\right)$. (2) and (7) then follow from the fact that $L_{l}\left(g_{1}+g_{2}\right)$ $\leqq L_{u}\left(g_{1}+g_{2}\right)$.

(4) and (5) follow from the inequality

$$
h-f \geqq(h \wedge x)-(f \wedge x) \text {. }
$$

(11) follows from the fact that every $g \in C^{*}$ is covered by an $h \in C$, and that 3.5 does not depend on (11).

4.7. $C^{*}$ is complete, in the sense that the process of extension described in 4.1-4.3 does not yield any new functions when applied to $C^{*}$.

Proof. It follows from 4.2 and 4.4 that

$$
\sup \left\{L^{*}(f) \mid f \in C^{*}, f \leqq g\right\}=\sup \{L(f) \mid f \in C, f \leqq g\},
$$

and similarly for the approximations from above.

4.8. Let $C$ be any module. Given $f \in C$ and a number $a>0$, let $1_{a}$ be the characteristic function of the set $\{x \mid f(x) \geqq a\}$. For each $f \in C$ there exists an everywhere dense set $S$ of real numbers $a>0$ such that $a \in S \rightarrow 1_{a} \in C^{*}$, where $C^{*}$ is the completion of $C$. Since $C^{*}$ is a module and is its own completion we have as a corollary the same theorem with the weaker assumption $f \in C^{*}$.

Proof. We shall prove the stronger result that there is at most a countable set $\left\{a_{i}\right\}$ of numbers $a_{i}>0$ such that $1_{a_{i}}$ is not in $C^{*}$. Given $e>0$, consider any $a \geqq e$ and numbers $b, c>0$ with $c \leqq e$. For any $d \geqq 0$ let $f^{d}=f \wedge d \in C$. Let $\phi(d)=L\left(f^{d}\right)$. We have 


$$
c^{-1}\left\{f^{a}-f^{a-c}\right\} \geqq 1_{a} \geqq b^{-1}\left\{f^{a+b}-f^{a}\right\},
$$

as may be seen by analyzing the three cases

$$
f(x) \geqq a+b, \quad a \leqq f(x)<a+b, \quad f(x)<a .
$$

Using 4.6 and (7), (8) of 3.1 , we have from (1) that

(2) $c^{-1}[\phi(a)-\phi(a-c)] \geqq L_{u}\left(1_{a}\right) \geqq L_{l}\left(1_{a}\right) \geqq b^{-1}[\phi(a+b)-\phi(a)]$.

The outside inequalities imply that $\phi(a)$ is a convex function for $a \geqq e$. Taking limits in (2) as $b, c \rightarrow 0$, we have further that

$$
D^{-} \phi(a) \geqq L_{u}\left(1_{a}\right) \geqq L_{l}\left(1_{a}\right) \geqq D^{+} \phi(a) .
$$

Since $\phi(a)$ is convex in the interval in question, $D^{-} \phi \neq D^{+} \phi$ at most at a countable number of points $\left\{a_{i}^{\prime}\right\}, a_{i}^{\prime} \geqq e$. Hence when $a \geqq e$ is not in $\left\{a_{i}^{\prime}\right\}, D^{-} \phi=D^{+} \phi$ and $1_{a} \in C^{*}$ by 4.3. By taking successively $e=1 / n, n=1,2, \cdots$, we get at most a countable sum of countable sets-that is, at most a countable set $\left\{a_{i}\right\}$-in the interval $a>0$ such that $1_{a_{i}}$ is not in $C^{*}$.

4.9. Let $\mathbf{A}^{*}=\left\{A \mid 1_{A} \in C^{*}\right\}$. Then

$$
f \in C^{*} \rightarrow L^{*}(f)=\lim L^{*}\left(f_{A}\right),
$$

where the limit is the limit taken on the directed system $A^{*}$ ordered by $\supset$.

Proof. It is sufficient to prove 4.9 for $f \geqq 0$. By 4.8 there exists a sequence $a_{n} \downarrow 0$ such that the characteristic functions $1_{n}$ of the sets $\left\{x \mid f(x) \geqq a_{n}\right\}$ are all in $C^{*}$. Put $g_{n}=f-\left(f \wedge a_{n}\right)$. Then $g_{n} \in C^{*}, g_{n} \leqq f$, and $g_{n} \cdot 1_{n}=g_{n}$. Hence

$$
L^{*}\left(g_{n}\right) \leqq L^{*}\left(f \cdot 1_{n}\right) \leqq L^{*}(f) .
$$

But inf $L^{*}\left(f-g_{n}\right)=0$ by 3.1 , (11), 4.9 for $f \geqq 0$ now follows, since if $f \geqq 0$

$$
\begin{aligned}
L^{*}(f) \geqq \lim _{\boldsymbol{A}} L^{*}\left(f_{A}\right) & =\sup \left\{L^{*}\left(f_{A}\right) \mid A \in \mathrm{A}^{*}\right\} \\
& \geqq \sup _{n} L^{*}\left(f \cdot 1_{n}\right) \geqq \sup _{n} L^{*}\left(g_{n}\right)=L^{*}(f) .
\end{aligned}
$$

\section{Extension of $L^{*}$ to "unbounded" functions.}

5.1. $C^{* *} \equiv\left\{f \mid A \in \mathrm{A}^{*} \rightarrow f_{A} \in C^{*}\right\}$.

5.2(a). $L^{* *}(f) \equiv \lim _{A} L^{*}\left(f_{A}\right)$ (for $f \in C^{* *}, f \geqq 0$ ). Here the limit is taken as in 4.9 .

5.2. $L^{* *}(f) \equiv L^{* *}\left(f^{+}\right)-L^{* *}\left(f^{-}\right)=\lim _{A} L^{*}\left(f_{A}^{+}\right)-\lim _{A} L^{*}\left(f_{A}^{-}\right)=\lim _{A} L^{*}\left(f_{A}\right)$ (for $f \in C^{* *}$ and $\left.L^{* *}\left(f^{+}\right), L^{* *}\left(f^{-}\right)<\infty\right)$. Thus $\left|L^{* *}(f)\right|<\infty$, except possibly if $f>0$. 
5.3. $C^{*} \subset C^{* *}$ and $f \in C^{*} \rightarrow L^{* *}(f)=L^{*}(f)(3.6,4.9)$.

5.4. $f \geqq 0 \rightarrow L^{* *}(f) \geqq 0$.

5.5. $f, g \in C^{* *} \rightarrow a f+b g \in C^{* *}$ and (if $\left.L^{* *}(f), L^{* *}(g)<\infty\right) L^{* *}(a f+b g)$ $=a L^{* *}(f)+b L^{* *}(g)$, since $(a f+b g) \cdot 1_{A}=a \cdot f 1_{A}+b \cdot g 1_{A}$, and $L^{* *}$ is defined as a limit on the directed set $\mathbf{A}^{*}$.

5.6. $f \leqq g \rightarrow L^{* *}(f) \leqq L^{* *}(g)(5.4,5.5)$.

5.7. $0 \leqq f \leqq g$ and $g \in C^{* *}$ and $L^{* *}(g)=0 \rightarrow f \in C^{* *}$ and $L^{* *}(f)=0$. For $f_{A} \in C^{*}$ by 4.7 . Hence by $5.4,5.6$

$$
f \in C^{* *}, \text { and } L^{* *}(f)=0
$$

5.8. $f, 1_{E} \in C^{* *} \rightarrow f_{E} \in C^{* *}$. For $1_{A} \in C^{*} \rightarrow\left(f_{E}\right) 1_{A}=\left(f_{A}\right)\left(1_{E} 1_{A}\right) \in C^{*}$ by 3.6 .

5.9. $f \in C^{* *}, f \geqq 0 \rightarrow L^{* *}(f)=\sup \left\{L^{* *}\left(f_{A}\right) \mid 1_{A} \in C\right\}(5.6,4.9,5.3)$.

5.10. $1_{X}=1 \in C^{* *}(5.1)$.

Actually 5.8 is a special case of the following theorem, which however will not be needed for this paper:

\subsection{1. $f, g \in C^{* *} \rightarrow f \cdot g \in C^{* *}$.}

Proof. Assume $0 \leqq h, i \in C^{*}$ and $1_{A} \in C^{*}$. It follows from 4.8 that we may subdivide $X$ into a finite number of sets $E_{\nu}$ such that $1_{E_{\nu}} \cdot 1_{A} \in C^{*}$ and that the oscillation of $h$ and $i$ on each set is less than $e$. Denoting by $a_{\nu}^{\prime \prime}, b_{\nu}^{\prime \prime}, a_{\nu}^{\prime}, b_{\nu}^{\prime}$ the maximum and minimum of $h$ and $i$ on $E_{\nu}$ we have

$$
\sum a_{\nu}^{\prime} \cdot b_{\nu} \cdot 1_{E_{\nu}} \cdot 1_{A} \leqq h \cdot i \cdot 1_{A} \leqq \sum a_{\nu}{ }^{\prime \prime} \cdot b_{\nu}^{\prime \prime} \cdot 1_{E_{\nu}} \cdot 1_{A} \cdot
$$

Hence by the completeness of $C^{*}: h \cdot i \cdot 1_{A} \in C^{*}$. The theorem now follows since putting $f_{A}=h$ and $g_{h}=i$ we have that $f \cdot g \cdot 1_{A}=f_{A} \cdot g_{A} \in C^{*}$ for every $1_{A} \in C^{*}$.

6. The $R$-measure defined by $L^{* *}$.

6.1(a). $\mathrm{A} \equiv\left\{E \mid 1_{E} \in C^{* *}\right\}$.

6.1(b). $V(E) \equiv L^{* *}\left(1_{E}\right)$ (for $E \in \mathrm{A}$ ).

6.1(c). $\mathbf{A}^{\prime} \equiv\{E \mid E \in \mathbf{A}$ and $V(E)<\infty\}$.

From these definitions it follows:

6.2. $V$ is an $R$-measure as defined in $\$ 1$.

Proof. The properties (1)-(5) are obvious from $\$ 5$. As for (6), we have from 3.1 (10) an $f \in C$ with $L(f)>0$. We can assume $0 \leqq f(x) \leqq 1$. If $L^{*}\left(f 1_{A}\right)=0$ for all $1_{A} \in C^{*}$, then $L(f)=0$ by $4.9,4.5$. Hence for one $1_{A}, L^{*}\left(f_{A}\right)>0$. But $1_{A} \geqq f_{A}$. Hence $L^{*}\left(1_{A}\right)=V(A)>0$.

7. Comparison of $L^{* *}(f)$ and $\int f(x) d V$.

7.1. $f \in C^{*}, f=f_{E} \geqq 0, E \in \mathrm{A}^{\prime} \rightarrow f \in R_{E}$ and $\int f=L^{*}(f)$.

Proof. (a) If $V(E)=0$, then $S_{u}\left(f_{E}\right)=S_{l}\left(f_{E}\right)=0=\int f$, since for some 
$a \neq 0,0 \leqq a f^{+} \leqq 1_{E}$ and $0 \leqq a f^{-} \leqq 1_{E}$, we have $L^{*}(a f)=0$ by $5.3,5.7$. Hence $L^{*}(f)=0=\int f$.

(b) If $0<V(E)<\infty$, given $e>0$, by 3.1 (1) and 4.8, there exists a partition $\delta$ of $X$ into sets $A_{0}, \cdots, A_{n} \in$ A such that

$$
\sup \left\{f(x)-f(y) \mid x, y \in A_{i}\right\}<e[V(E)]^{-1}, \quad i=0, \cdots, n .
$$

Let $E_{i}=E A_{i}$. Then $1_{E_{i}}=1_{i} \in C^{* *}$ and hence $f_{i}=f 1_{i} \in C^{* *}$ by 5.8 . Letting $b_{i}=\sup \left\{f(x) \mid x \in E_{i}\right\}$, we have

and

$$
L^{*}(f)=L^{* *}\left(f_{E}\right)=\sum_{i} L^{* *}\left(f 1_{i}\right)
$$

$$
S_{u}(f, E, \delta)=\sum_{i} V\left(E_{i}\right) b_{i}=\sum_{i} L^{* *}\left(1_{i} \cdot b_{i}\right)
$$

Hence $\left|L^{*}(f)-S_{u}(f, E, \delta)\right|<e$ and $L^{*}(f)=S_{u}(f, E)$. Similarly $L^{*}(f)=S(f, E)$.

7.2. If $f \geqq 0$, if $f \in R_{A}$ for $A \in A^{*}$, and if $f$ is bounded on any $E \in \mathbf{A}$, then

(a) $f \in R_{E}$ for every $E \in \mathrm{A}^{\prime}$,

(b) $\sup \left\{\int f_{E} \mid E \in \mathbf{A}^{\prime}\right\}=\sup \left\{\int f_{A} \mid A \in \mathbf{A}^{*}\right\}$.

Proof. Since $f$ is bounded on $E$ and $L^{* *}\left(1_{E}\right)<\infty, S_{u}(f, E)-S_{u}(f, A)$ $<e / 2$ for some $1_{A_{1}} \in C^{*}, 1_{A_{1}} \leqq 1_{E}$. Dually $S_{l}(f, E)-S_{l}\left(f, A_{2}\right)<e / 2$ $\left(1_{A_{2}} \in C^{*}, 1_{A_{2}} \leqq 1_{E}\right)$. The inequalities still hold if we replace $A_{1}$ and $A_{2}$ by $A=A_{1} \cup A_{2}$. Since $S_{u}(f, A)=S_{l}(f, A)$ we have $S_{u}(f, E)-S_{l}(f, E)<e$ for any $e$. Hence $f \in R_{E}$ and $\int f_{E}-\int f_{A}<e$ from which (b) follows.

7.3. $f \in C^{*} \rightarrow f \in R$ and $\int f=L^{*}(f)$.

Proof. Assume $f \geqq 0$. By 7.1, $f_{A} \in R_{A}$, that is, $f \in R_{A}$ for any $A \in \mathrm{A}^{*}$ and $\int f_{A}=L^{*}\left(f_{A}\right)$. Since $f$ is bounded, $f \in R_{E}\left(E \in \mathrm{A}^{\prime}\right)$ by 7.2(a). From $7.2(\mathrm{~b})$ and 4.9 it follows that $\sup \left\{\int f_{E} \mid E \in \mathrm{A}^{\prime}\right\}$ is equal to $L^{*}(f)$, hence finite, and equal to $\int f$ by 2.9. For any $f \in C^{*}, 7.3$ then follows by $2.91,2.92$.

7.4. $f \in R_{A}$ and $A \in \mathrm{A}^{*} \rightarrow f \in C^{*}$.

Proof. $f \in R_{A}$ means that $f$ can be approximated from above and below by functions $\sum a_{\nu} 1_{A_{\nu}}$ where $A_{\nu} \in A^{*}$. Hence $f \in C^{*}$ by 4.7 .

7.5. $f \in R \rightarrow f \in C^{* *}$ and $L^{* *}(f)=\int f$.

Proof. Assume $f \geqq 0$, then $f \in R \rightarrow f_{A} \in R_{A}$ for every $A \in \mathrm{A}^{*}$. By 7.4, $f_{A} \in C^{*}$ and hence $f \in C^{* *}$.

Furthermore $\int f=\sup \left\{\int f_{A} \mid A \in \mathbf{A}^{*}\right\}=\sup \left\{L^{*}\left(f_{A}\right) \mid A \in \mathbf{A}^{\prime}\right\}$ $=L^{* *}(f)(2.9,7.2,7.1)$. For any $f \in R, 7.5$ follows from its truth for $f^{+}, f^{-}$.

The proof of our main theorem, 3.2, is now complete: That $V$ is 
an $R$-measure was shown in $\$ 6,(1)$ and (2) follow from 7.3 , (3) from 7.5 and the definition of $L^{* *}$.

8. Some special cases.

A. 8.1. Assume that $f(x)=1 \in C$ and $L(1)=1$. (This makes 3.1 (5), (10) and (11) redundant, (11) follows from the fact that $L(f \wedge a)$ $\leqq L(a \cdot 1)=a$.) In this case $V(X)=1$ and 3.2 reduces to Bochner's theorem.

B. 8.2. Definition. By an $L$-extension of $V$ we shall mean a countably additive, complete measure $U$ defined for sets of a countably additive, complemented family $\mathbf{B}$ such that $\mathbf{B} \supset \mathbf{A}$ and for $E \in \mathbf{A}$, $U(E)=V(E)$.

8.3. Replace $3.1(11)$ by $3.1(12):\left\{f_{n}, g \in C, 0 \leqq f_{n} \leqq g, \lim _{n} f_{n}(x)=0\right.$ for all $x\} \rightarrow \lim _{n} L\left(f_{n}\right)=0$. Then (a) Theorem 3.2 still holds and in addition (b) $V$ possesses an $L$-extension $U$ such that (c) 3.2 (3) holds when " $g \in R$ " is replaced by " $g$ is measurable and integrable $(U)$."

Proof. (a) 3.1 (12) implies 3.1 (11). For $f \in C$ put $f_{n}=\inf (f, 1 / n)$. Then $\inf _{a>0} L(f \wedge a) \leqq \lim L\left(f_{n}^{+}\right)=0$.

(b) It is known ${ }^{2}$ that any $V$ with properties 1.1 (1)-(5) possesses an $L$-extension if and only if $V$ has the additional property: $\left\{E_{n} \in \mathbf{A}\right.$, $\left.E_{n} \geqq E_{n+1}, V\left(E_{1}\right)<\infty, \bigcap_{n} E_{n}=\varnothing\right\} \rightarrow \lim _{n} V\left(E_{n}\right)=0$. That $V$ has this property follows from

8.4. $\left\{f_{n} \in C^{* *}, f_{n} \geqq f_{n+1}\right.$, for each $x$ inf $\left.f_{n}(x)=0, L^{* *}\left(f_{1}\right)<\infty\right\}$ $\rightarrow \lim L^{* *}\left(f_{n}\right)=0$.

Proof. Suppose that for all $n, L^{* *}\left(f_{n}\right) \geqq e>0$. Since $L^{* *}\left(f_{n}\right)<\infty$ there exists an $A \in \mathrm{A}^{*}$ such that $L^{* *}\left(f_{1}-f_{1 A}\right) \leqq e / 2$. Since $\left(1-1_{A}\right) f_{n}$ $\leqq\left(1-1_{A}\right) f_{1}$ we also have $L^{* *}\left(f_{n}-f_{n A}\right) \leqq e / 2$, and $L^{* *}\left(f_{n A}\right) \geqq e / 2$ for all $n$. Hence we can find a $g_{n} \in C$ such that $0 \leqq g_{n} \leqq f_{n A}$ and $L\left(g_{n}\right) \geqq e / 3$. But evidently for each $x, \lim g_{n}(x)=0$. Since there exists a $g \in C$ such that $g \geqq f_{1} \cdot 1_{A}>g_{n}$, this contradicts 3.1 (12).

(c) To show that the analogue of 3.2 (3) holds for $g$ measurable and integrable $(U)$, we point out that (a) is sufficient to show that 3.2 (3) holds when $g$ is the characteristic function of a set $B$ measurable ( $U$ ) with $U(B)<\infty$ and (b) if $U$ is an $L$-extension of $V$ then, given $e>0, V$ contains an $E \in \mathrm{A}$ such that $V(E)>U(E)-e$. The result then follows from 3.2 (3).

(a) is a consequence of the ordinary Lebesgue theory while (b) results from the manner in which $U$ is defined as an extension of $V .^{2}$

Princeton University

${ }^{2}$ This theorem is proved by Kolmogoroff (A. Kolmogoroff, Grundbegriffe der Wahrscheinlichkeitsrechnung, Berlin, 1933) for the case $V(X)=1$. When $X$ is the sum of countably many sets of finite measure, the proof given by Jessen (B. Jessen, Abstrakt maal- og integraltheorie, 1, Matematisk Tidsskrift (B) (1934) p. 78) applies. The proof in the general case follows by a modification of that of Jessen. 